\title{
A Computational Solution of Natural Convection Flow in a Rotating Fluid with Radiative Heat Transfer
}

\author{
Harouna Naroua \\ Département de Mathématiques et Informatique, Faculté des Sciences et Techniques, Université Abdou \\ Moumouni, Niamey, Niger \\ Email: hnaroua@yahoo.com
}

Received 4 March 2016; accepted 10 June 2016; published 13 June 2016

Copyright @ 2016 by author and Scientific Research Publishing Inc.

This work is licensed under the Creative Commons Attribution International License (CC BY). http://creativecommons.org/licenses/by/4.0/

c) (i) Open Access

\section{Abstract}

An analysis of the hydromagnetic free convective flow past a vertical infinite porous plate in a rotating fluid is carried out. The temperatures involved are assumed to be very large so that the radiative heat transfer is significant, which renders the problem very non-linear even on the assumption of a differential approximation for the radiative flux. The temperature and velocity fields are computed using a generic software tool based on the Nakamura finite difference scheme. The genericity of the software tool is in the sense that it is a common solution to the category of time dependent laminar fluid flows expressed in one spatial coordinate. The input equations, together with other relevant parameters, are transformed into postfix code which will be farther interpreted in the computation process. The influence of the various parameters entering into the problem is shown graphically followed by a discussion of results.

\section{Keywords}

Computer Simulation, Computational Solution, Generic Software Tool, Laminar Fluid Flow

\section{Introduction}

Extensive research efforts have been directed to the study of the theory of rotating fluids due to its application in Cosmical and Geophysical fluid dynamics, meteorology and engineering [1]. Bachelor [2] studied the Eckman layer flow on a horizontal plate. The flow past a horizontal plate has also been studied by [3]-[6]. In all these investigations, the effects of radiative heat transfer have been ignored. However, high temperature phenomena abound in solar physics, particularly in astrophysical studies. Naroua [7] presented a numerical simulation on un- 
steady hydromagnetic free convection near a moving infinite flat plate in a rotating medium. The temperatures involved are assumed to be very high so that the radiative heat transfer is significant, which renders the problem highly non-linear even with the assumption of a differential approximation for the radiative heat flux. Yamauchi et al. [8] presented modified finite-difference formulas for a general proposition of an interface that they applied to the propagating beam analysis of z-variant rib waveguides. They observed that a discretization error is satisfactorily reduced in tilted and tapered rib waveguides. Zhu et al. [9] analyzed explicit/implicit schemes for parabolic equations with discontinuous coefficients. Numerical experiments, which were given for both linear and nonlinear problems, showed that their theoretical estimates were optimal in some sense. Khader and Ahmed [10] introduced a numerical simulation using finite difference method with the theoretical study for the problem of the flow and heat transfer over an unsteady stretching sheet embedded in a porous medium in the presence of a thermal radiation. Matsuoka and Nakamura [11] proposed a stable numerical scheme for a Cahn-Hilliard type equation with long-range interaction describing the micro-phase separation of diblock copolymer melts. They designed their scheme by using the discrete variational derivative method which is one of the structure preserving numerical methods. They observed that their proposed scheme has the same characteristic properties, mass conservation and energy dissipation, as the original equation does. They also discussed the stability and unique solvability of their proposed scheme.

This paper therefore incorporates radiative transfer into the study of natural convection in a rotating fluid thereby widening the applicability of the results. For an optically thin gas in a transparent medium with the absorption coefficient $\alpha(\alpha \gg 1)$ which will be assumed constant in the analysis, the radiative flux $Q^{\prime}$ satisfies the governing non-linear differential equation given by [12]:

$$
\frac{\partial Q^{\prime}}{\partial z^{\prime}}=4 \sigma \alpha\left(T^{\prime 4}-T_{\infty}^{\prime 4}\right)
$$

where $T^{\prime}$ is the temperature of the fluid; subscript $\infty$ will be used to denote conditions in the undisturbed fluid and $\sigma$ is the Stefan - Boltzmann constant.

\section{Mathematical Analysis}

Consider the unsteady convective flow of an electrically conducting incompressible viscous fluid past an infinite vertical porous flat plate at $z^{\prime}=0$. Let the fluid and the plate be in a state of rigid rotation with uniform angular velocity $\Omega$ about the $z^{\prime}$ axis which is normal to the plate. A uniform magnetic field $B_{0}$ is imposed along $z^{\prime}$-axis and the plate is assumed to be electrically non-conducting. The temperature of the plate is maintained at $T_{w}^{\prime}\left(T_{w}^{\prime} \gg 1\right)$. Since the plate is infinite in extent, all the physical variables depend on $z^{\prime}$ and $t^{\prime}$ only. In a rotating frame of reference employing Equation (1), the governing equations for a transparent medium are given by:

$$
\begin{aligned}
& \frac{\partial u^{\prime}}{\partial t^{\prime}}-w_{0} \frac{\partial u^{\prime}}{\partial z^{\prime}}-2 \Omega v^{\prime}=v \frac{\partial^{2} u^{\prime}}{\partial z^{2}}+g \beta\left(T^{\prime}-T_{\infty}^{\prime}\right)-\frac{\sigma_{c}}{\rho} B_{0}^{2} u^{\prime} \\
& \frac{\partial v^{\prime}}{\partial t^{\prime}}-w_{0} \frac{\partial v^{\prime}}{\partial z^{\prime}}+2 \Omega u^{\prime}=v \frac{\partial^{2} v^{\prime}}{\partial z^{2}}-\frac{\sigma_{c}}{\rho} B_{0}^{2} v^{\prime} \\
& \rho C_{p}\left(\frac{\partial T^{\prime}}{\partial t^{\prime}}-w_{0} \frac{\partial T^{\prime}}{\partial z^{\prime}}\right)=k \frac{\partial^{2} T^{\prime}}{\partial z^{\prime 2}}-4 \sigma \alpha\left(T^{\prime 4}-T_{\infty}^{\prime 4}\right)
\end{aligned}
$$

where $\left(u^{\prime}, v^{\prime}, 0\right)$ are the velocity components along $x^{\prime}, y^{\prime}$ and $z^{\prime}$ directions; $\rho$ is the density; $v$ is the kinematic coefficient of viscosity; $k$ is the thermal conductivity; $C_{p}$ is the specific heat of the fluid; $g$ is the acceleration due to gravity; $\beta$ is the coefficient of volume expansion; $\sigma_{c}$ is the electrical conductivity of the fluid and $w_{o}$ is the constant suction velocity.

The boundary conditions are given by:

$$
\begin{array}{ll}
u^{\prime}=0, v^{\prime}=0, T^{\prime}=T_{w}^{\prime} & \text { on } z^{\prime}=0 \\
u^{\prime}=0, v^{\prime}=0, T^{\prime}=T_{\infty}^{\prime} & \text { on } z^{\prime} \rightarrow \infty
\end{array}
$$

Introducing the following non-dimensional quantities 


$$
\begin{aligned}
& t=\frac{t^{\prime} w_{0}}{4 v}, z=\frac{z^{\prime} w_{0}}{v},(u, v)=\frac{\left(u^{\prime}, v^{\prime}\right)}{w_{0}},\left(\theta, \theta_{w}\right)=\frac{\left(T^{\prime}, T_{w}^{\prime}\right)}{T_{\infty}^{\prime}}, \operatorname{Er}=\frac{\Omega v}{w_{0}^{2}}, \\
& \operatorname{Pr}=\frac{\rho v C_{p}}{k}, M^{2}=\frac{\sigma_{c} B_{0}^{2} v}{\rho w_{0}^{2}}, G r=\frac{v g \beta T_{\infty}^{\prime}}{w_{0}^{3}}, R=\frac{4 \sigma \alpha v T_{\infty}^{\prime 3}}{\rho C_{p} w_{0}^{2}}
\end{aligned}
$$

Equations (2)-(5) reduce to:

$$
\left\{\begin{array}{l}
\frac{1}{4} \frac{\partial u}{\partial t}-\frac{\partial u}{\partial z}-2 E r \cdot v=\frac{\partial^{2} u}{\partial z^{2}}-M^{2} u+G r(\theta-1) \\
\frac{1}{4} \frac{\partial v}{\partial t}-\frac{\partial v}{\partial z}+2 E r \cdot u=\frac{\partial^{2} v}{\partial z^{2}}-M^{2} v \\
\frac{\operatorname{Pr}}{4} \frac{\partial \theta}{\partial t}-\operatorname{Pr} \frac{\partial \theta}{\partial z}=\frac{\partial^{2} \theta}{\partial z^{2}}-R\left(\theta^{4}-1\right)
\end{array}\right.
$$

where

$$
\begin{cases}u=0, \quad v=0, \quad \theta=\theta_{w} & \text { at } z=0 \\ u \rightarrow 0, \quad v \rightarrow 0, \quad \theta \rightarrow 0 & \text { as } z \rightarrow \infty\end{cases}
$$

The above system of Equations (7)-(9) with boundary conditions (10) has been solved numerically by a generic software based on the Nakamura [13] scheme. Its' genericity is in providing a common solution to the category of time dependent laminar fluid flow problems expressed in one spatial coordinate. It may be one- dimensional, two-dimensional or three-dimensional fluid flows. The mesh system is shown in Figure 1.

Equations (7)-(9) are coupled non-linear parabolic partial differential equations in $u, v$ and $\theta$. First, $\frac{\partial u}{\partial t}, \frac{\partial v}{\partial t}$ and $\frac{\partial \theta}{\partial t}$ are transformed using the backward difference approximation (which is stable) as shown in Equations

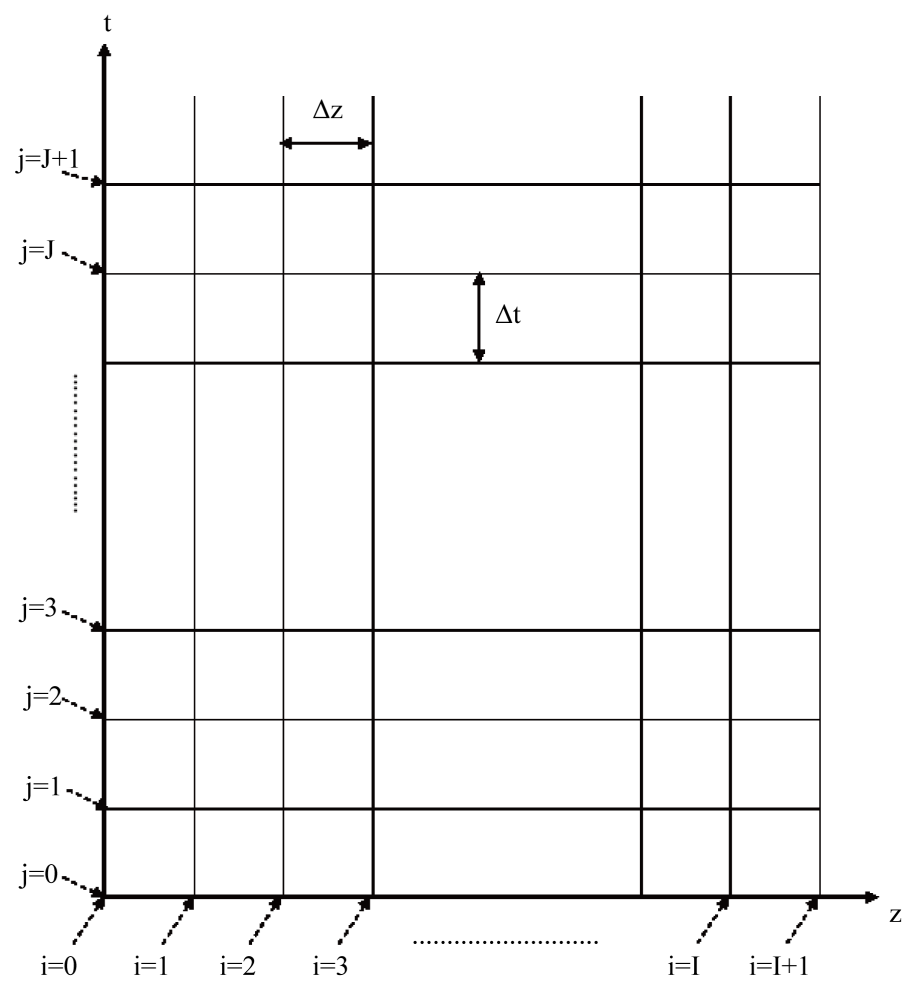

Figure 1. Mesh system. 
(11)-(13):

$$
\left\{\begin{array}{l}
u^{\prime \prime}+u^{\prime}-\left(M^{2}+\frac{1}{4 \Delta t}\right) u=-\frac{1}{4 \Delta t} u_{i, j-1}-2 E r \cdot v-G r(\theta-1) \\
v^{\prime \prime}+v^{\prime}-\left(M^{2}+\frac{1}{4 \Delta t}\right) v=-\frac{1}{4 \Delta t} v_{i, j-1}+2 E r \cdot u \\
\theta^{\prime \prime}+\operatorname{Pr} \theta^{\prime}-\left(R \theta^{3}+\frac{P r}{4 \Delta t}\right) \theta=-\frac{\operatorname{Pr}}{4 \Delta t} \theta_{i, j-1}-R
\end{array}\right.
$$

where $u^{\prime}, u^{\prime \prime}, v^{\prime}, v^{\prime \prime}, \theta^{\prime}, \theta^{\prime \prime}$ are derivatives with respect to $z$.

For the sake of simplicity, we write:

$$
\begin{aligned}
& B_{2}=1 ; B_{1}=1 ; B_{0}=-\left(M^{2}+\frac{1}{4 \Delta t}\right) ; P_{i, j-1}^{1}=-\frac{1}{4 \Delta t} u_{i, j-1}-2 E r \cdot v-G r(\theta-1) ; \\
& C_{2}=1 ; C_{1}=1 ; C_{0}=-\left(M^{2}+\frac{1}{4 \Delta t}\right) ; P_{i, j-1}^{2}=-\frac{1}{4 \Delta t} v_{i, j-1}+2 E r \cdot u \\
& D_{2}=1 ; D_{1}=\operatorname{Pr} ; D_{0}=-\left(R \theta^{3}+\frac{P r}{4 \Delta t}\right) ; P_{i, j-1}^{3}=-\frac{P r}{4 \Delta t} \theta_{i, j-1}-R
\end{aligned}
$$

Using the above formulation, Equations (11)-(13) take the form:

$$
\left\{\begin{array}{l}
B_{2} u_{i, j}^{\prime \prime}+B_{1} u_{i, j}^{\prime}+B_{0} u_{i, j}=P_{i, j-1}^{1} \\
C_{2} v_{i, j}^{\prime \prime}+C_{1} v_{i, j}^{\prime}+C_{0} v_{i, j}=P_{i, j-1}^{2} \\
D_{2} \theta_{i, j}^{\prime \prime}+D_{1} \theta_{i, j}^{\prime}+D_{0} \theta_{i, j}=P_{i, j-1}^{3}
\end{array}\right.
$$

Using the central difference scheme which is unconditionally stable, Equations (14)-(16) reduce to:

$$
\left\{\begin{array}{l}
B_{2}\left(\frac{u_{i+1, j}-2 u_{i, j}+u_{i-1, j}}{(\Delta z)^{2}}\right)+B_{1}\left(\frac{u_{i+1, j}-u_{i-1, j}}{2 \Delta z}\right)+B_{0} u_{i, j}=P_{i, j-1}^{1} \\
C_{2}\left(\frac{v_{i+1, j}-2 v_{i, j}+v_{i-1, j}}{(\Delta z)^{2}}\right)+C_{1}\left(\frac{v_{i+1, j}-v_{i-1, j}}{2 \Delta z}\right)+C_{0} v_{i, j}=P_{i, j-1}^{2} \\
D_{2}\left(\frac{\theta_{i+1, j}-2 \theta_{i, j}+\theta_{i-1, j}}{(\Delta z)^{2}}\right)+D_{1}\left(\frac{\theta_{i+1, j}-\theta_{i-1, j}}{2 \Delta z}\right)+D_{0} \theta_{i, j}=P_{i, j-1}^{3}
\end{array}\right.
$$

At time step $j+1$, Equations (17)-(19) reduce to:

$$
\left\{\begin{array}{l}
\left(\frac{B_{2}}{(\Delta z)^{2}}-\frac{B_{1}}{2 \Delta z}\right) \cdot u_{i-1, j+1}+\left(B_{0}-\frac{2 B_{2}}{(\Delta z)^{2}}\right) \cdot u_{i, j+1}+\left(\frac{B_{2}}{(\Delta z)^{2}}+\frac{B_{1}}{2 \Delta z}\right) \cdot u_{i+1, j+1}=P_{i, j}^{1} \\
\left(\frac{C_{2}}{(\Delta z)^{2}}-\frac{C_{1}}{2 \Delta z}\right) \cdot v_{i-1, j+1}+\left(C_{0}-\frac{2 C_{2}}{(\Delta z)^{2}}\right) \cdot v_{i, j+1}+\left(\frac{C_{2}}{(\Delta z)^{2}}+\frac{C_{1}}{2 \Delta z}\right) \cdot v_{i+1, j+1}=P_{i, j}^{2} \\
\left(\frac{D_{2}}{(\Delta z)^{2}}-\frac{D_{1}}{2 \Delta z}\right) \theta_{i-1, j+1}+\left(D_{0}-\frac{2 D_{2}}{(\Delta z)^{2}}\right) \cdot \theta_{i, j+1}+\left(\frac{D_{2}}{(\Delta z)^{2}}+\frac{D_{1}}{2 \Delta z}\right) \cdot \theta_{i+1, j+1}=P_{i, j}^{3}
\end{array}\right.
$$

If the problem is well defined, Equations (20)-(22) admit a solution but cannot be solved individually for each grid point $i$. The equations for all the grid points must be solved simultaneously. The set of equations for $i=1,2, \cdots, I$ forms a tridiagonal system of equations as described by Nakamura [13] and shown in Equations (23)-(25). 
H. Naroua

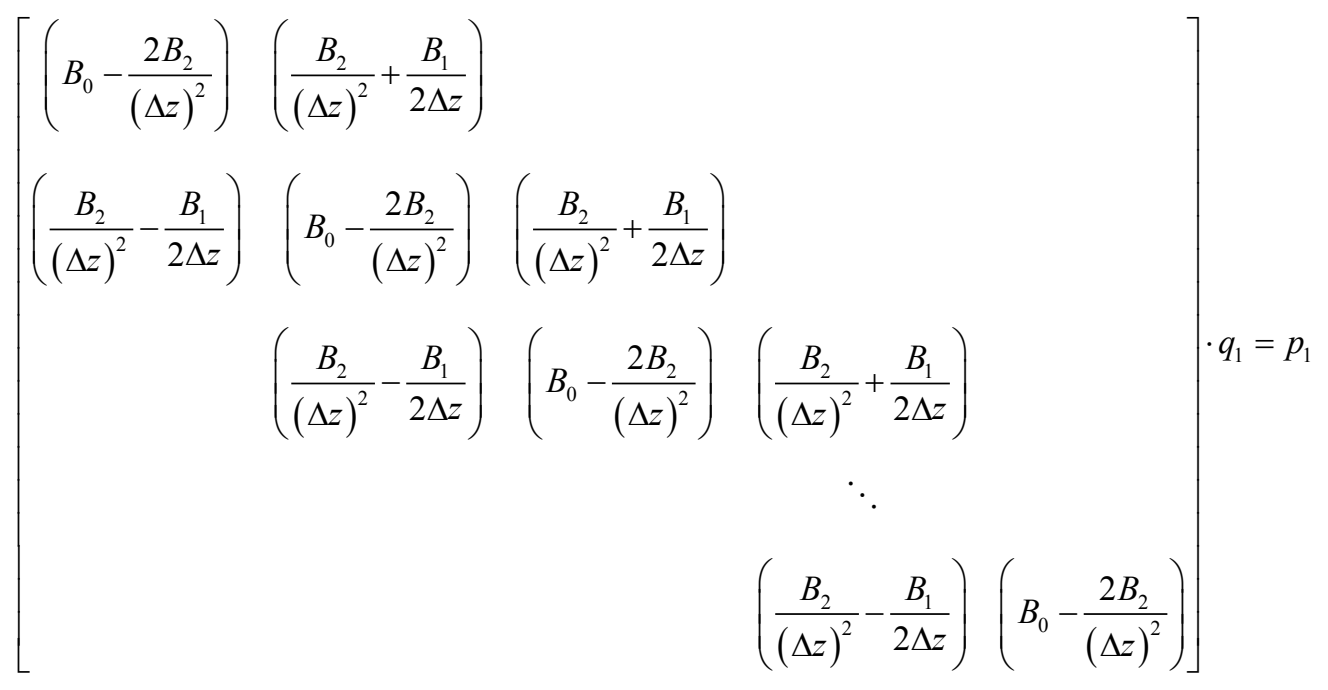

where $q_{1}=\left[\begin{array}{c}u_{1, j+1} \\ u_{2, j+1} \\ u_{3, j+1} \\ \vdots \\ u_{i, j+1} \\ \vdots \\ u_{I, j+1}\end{array}\right]$ and $p_{1}=\left[\begin{array}{c}P_{1, j}^{1} \\ P_{2, j}^{1} \\ P_{3, j}^{1} \\ \vdots \\ P_{i, j}^{1} \\ \vdots \\ P_{I, j}^{1}\end{array}\right]$

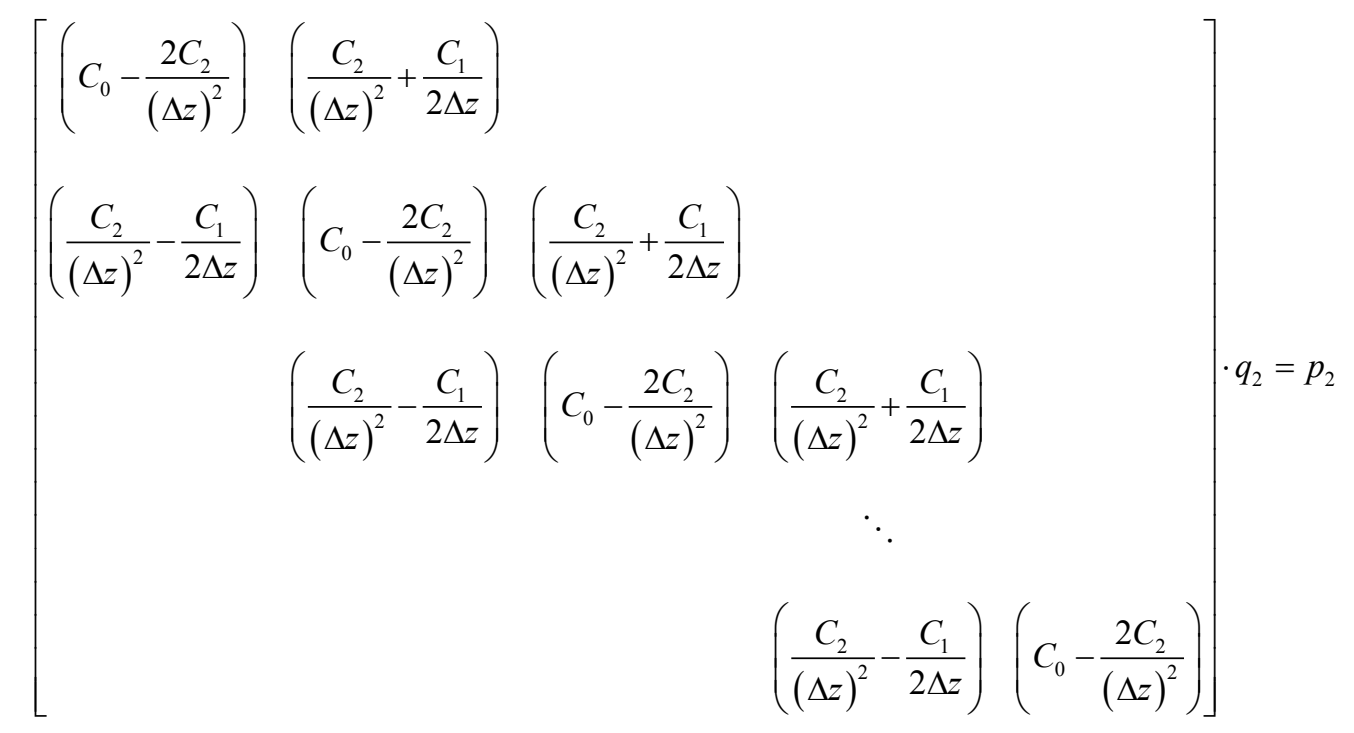

where $q_{2}=\left[\begin{array}{c}v_{1, j+1} \\ v_{2, j+1} \\ v_{3, j+1} \\ \vdots \\ v_{i, j+1} \\ \vdots \\ v_{I, j+1}\end{array}\right]$ and $p_{2}=\left[\begin{array}{c}P_{1, j}^{2} \\ P_{2, j}^{2} \\ P_{3, j}^{2} \\ \vdots \\ P_{i, j}^{2} \\ \vdots \\ P_{I, j}^{2}\end{array}\right]$ 


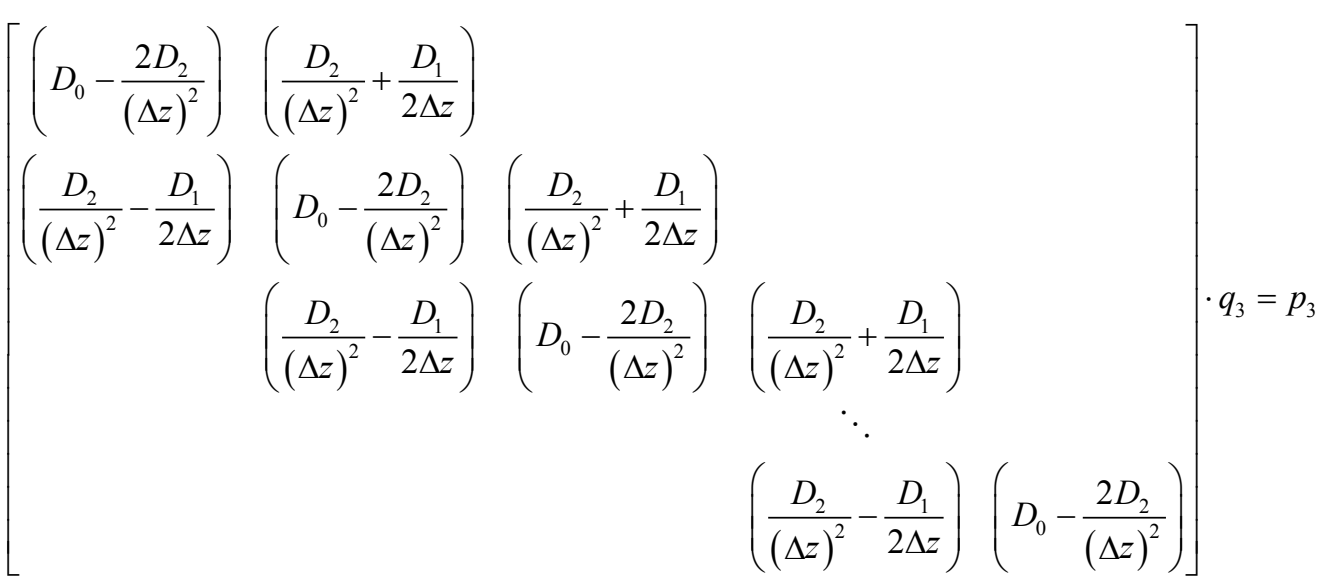

where $q_{3}=\left[\begin{array}{c}\theta_{1, j+1} \\ \theta_{2, j+1} \\ \theta_{3, j+1} \\ \vdots \\ \theta_{i, j+1} \\ \vdots \\ \theta_{I, j+1}\end{array}\right]$ and $p_{3}=\left[\begin{array}{c}P_{1, j}^{3} \\ P_{2, j}^{3} \\ P_{3, j}^{3} \\ \vdots \\ P_{i, j}^{3} \\ \vdots \\ P_{I, j}^{3}\end{array}\right]$

For each time step, the system of Equations (23)-(25) requires an iterative procedure due to the presence of non-linear coefficients. Successive substitution and iteration are continuously executed for each time step until convergence is reached.

\section{Discussion of Results}

In order to investigate the behavior of the velocity and temperature profiles, curves are drawn for various values of the parameters that describe the flow and are displayed in Figures 2-11.

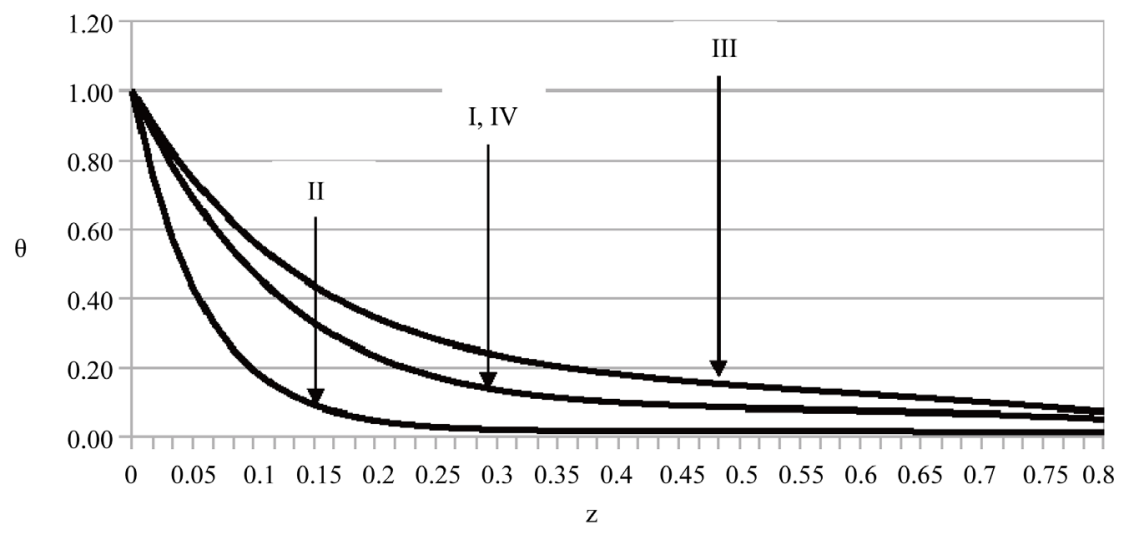

\begin{tabular}{|c|c|c|c|}
\hline Series & Pr & Er & R \\
\hline I & 0.71 & 0.001 & 3 \\
\hline II & 7 & 0.001 & 3 \\
\hline III & 0.71 & 0.005 & 3 \\
\hline IV & 0.71 & 0.001 & 30 \\
\hline
\end{tabular}

Figure 2. Transient temperature profiles $(\theta)$. 


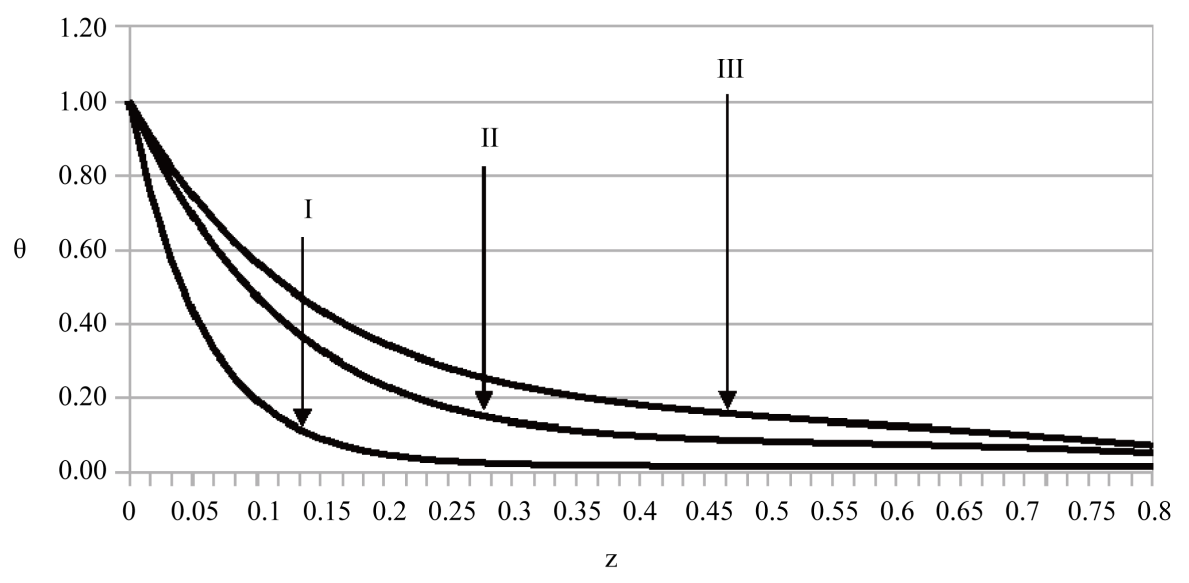

\begin{tabular}{|c|c|}
\hline Series & t \\
\hline I & 0.01 \\
\hline II & 0.10 \\
\hline III & 0.50 \\
\hline
\end{tabular}

Figure 3. Transient temperature profiles $(\theta)$.

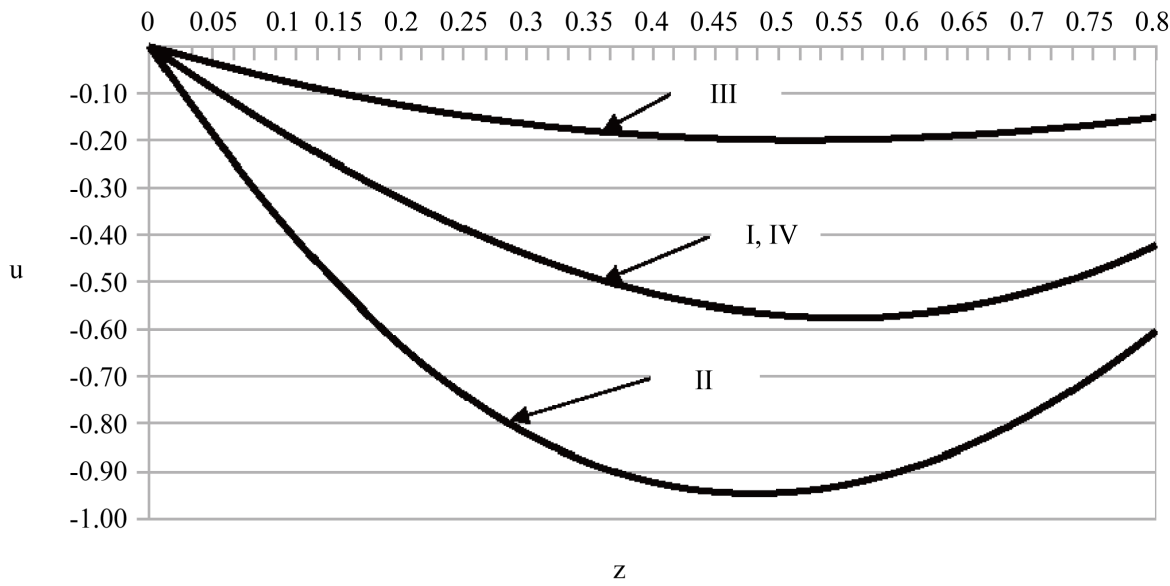

\begin{tabular}{|c|c|c|c|}
\hline Series & $\boldsymbol{P r}$ & $\boldsymbol{E} \boldsymbol{r}$ & $\boldsymbol{R}$ \\
\hline I & 0.71 & 0.001 & 3 \\
\hline II & 7 & 0.001 & 3 \\
\hline III & 0.71 & 0.005 & 3 \\
\hline IV & 0.71 & 0.001 & 30 \\
\hline
\end{tabular}

Figure 4. Transient velocity profiles $(u)$ for $G r=10$.

From Figure 2 and Figure 3, it is observed that:

1) the temperature profile $(\theta)$ increases due to an increase in time $(t)$;

2) there is a fall in temperature profile $(\theta)$ due to an increase in Prandtl number $(\operatorname{Pr})$;

3 ) there is a rise in temperature profile $(\theta)$ due to an increase in rotation parameter $(E r)$;

$4)$ there is an insignificant change in the temperature profile $(\theta)$ due to an increase in radiation parameter $(R)$. 


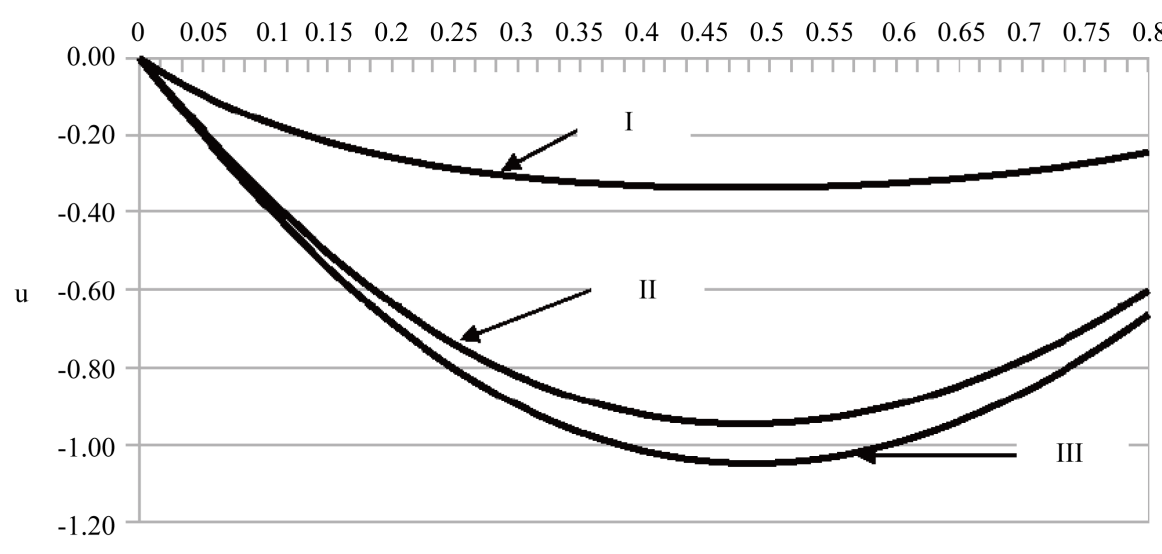

Z

\begin{tabular}{|c|c|}
\hline Series & t \\
\hline I & 0.01 \\
\hline II & 0.10 \\
\hline III & 0.50 \\
\hline
\end{tabular}

Figure 5. Transient velocity profiles $(u)$ for $G r=10$.

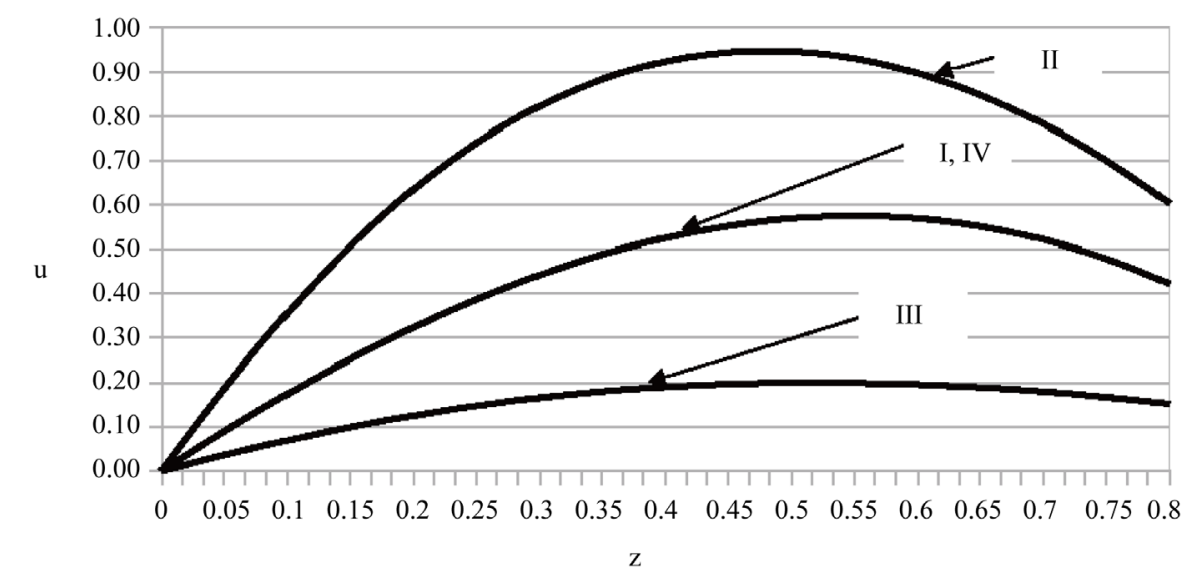

\begin{tabular}{|c|c|c|c|}
\hline Series & $\boldsymbol{P r}$ & $\boldsymbol{E} \boldsymbol{r}$ & $\boldsymbol{R}$ \\
\hline I & 0.71 & 0.001 & 3 \\
\hline II & 7 & 0.001 & 3 \\
\hline III & 0.71 & 0.005 & 3 \\
\hline IV & 0.71 & 0.001 & 30 \\
\hline
\end{tabular}

Figure 6. Transient velocity profiles $(u)$ for $G r=-10$.

From Figure 4 and Figure 5, for $G r>0$ (in the presence of cooling of the plate by free convection currents) it is observed that:

1) the transient primary velocity profile $(u)$ is backward;

2) the transient primary velocity profile $(u)$ decreases due to an increase in time $(t)$;

$3)$ there is a rise in the transient primary velocity profiles $(u)$ due to an increase in rotation parameter $(E r)$ whereas the transient velocity field $(u)$ decreases due to an increase in Prandtl number $(\operatorname{Pr})$; 


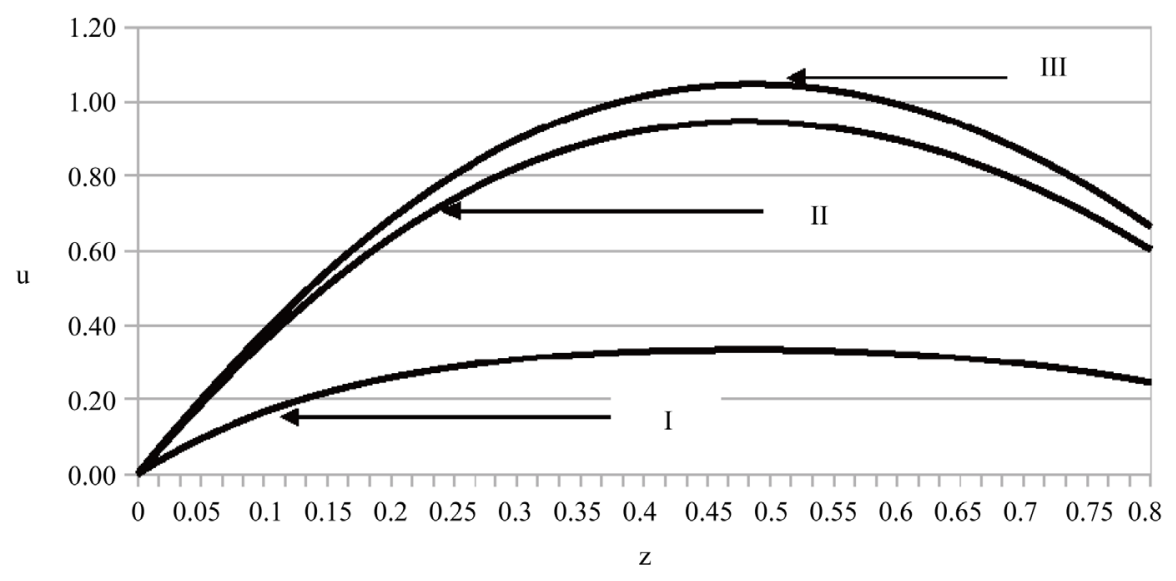

\begin{tabular}{|c|c|}
\hline Series & t \\
\hline I & 0.01 \\
\hline II & 0.10 \\
\hline III & 0.50 \\
\hline
\end{tabular}

Figure 7. Transient velocity profiles $(u)$ for $G r=-10$.

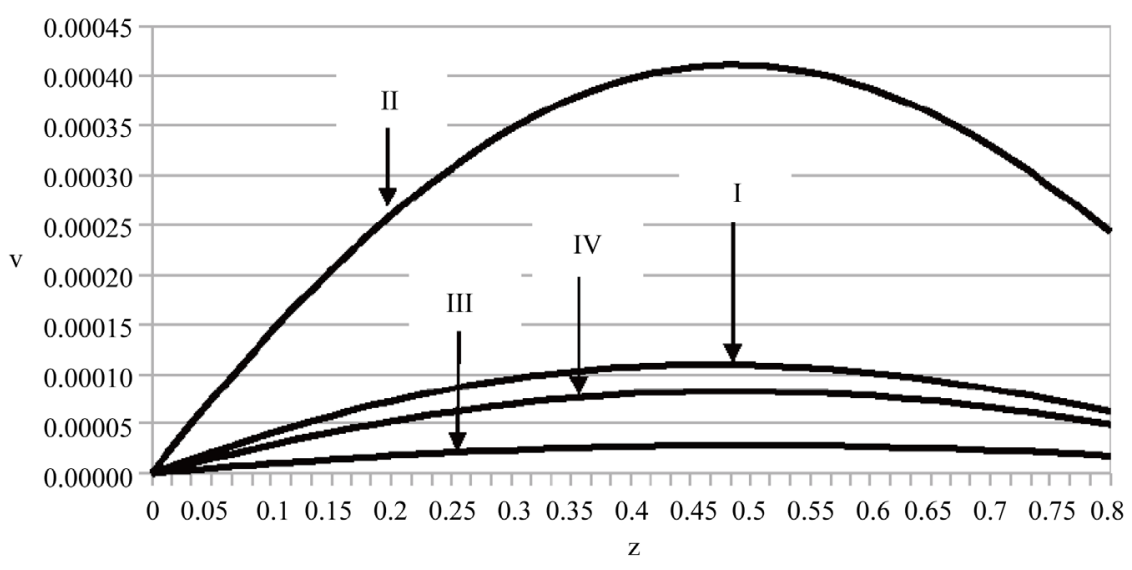

\begin{tabular}{|c|c|c|c|}
\hline Series & Pr & Er & R \\
\hline I & 0.71 & 0.001 & 3 \\
\hline II & 7 & 0.001 & 3 \\
\hline III & 0.71 & 0.005 & 3 \\
\hline IV & 0.71 & 0.001 & 30 \\
\hline
\end{tabular}

Figure 8. Transient velocity profiles (v) for $G r=10$.

4) there is an insignificant change in the transient primary velocity profile $(u)$ due to an increase in radiation parameter $(R)$.

From Figure 6 and Figure 7, for $\mathrm{Gr}<0$ (in the presence of heating of the plate by free convection currents) it is observed that:

1) the transient primary velocity profile $(u)$ increases due to an increase in time $(t)$;

$2)$ there is a fall in the primary velocity profile $(u)$ due to an increase in rotation parameter $(E r)$ whereas u rises with an increase in Prandtl number $(\mathrm{Pr})$; 


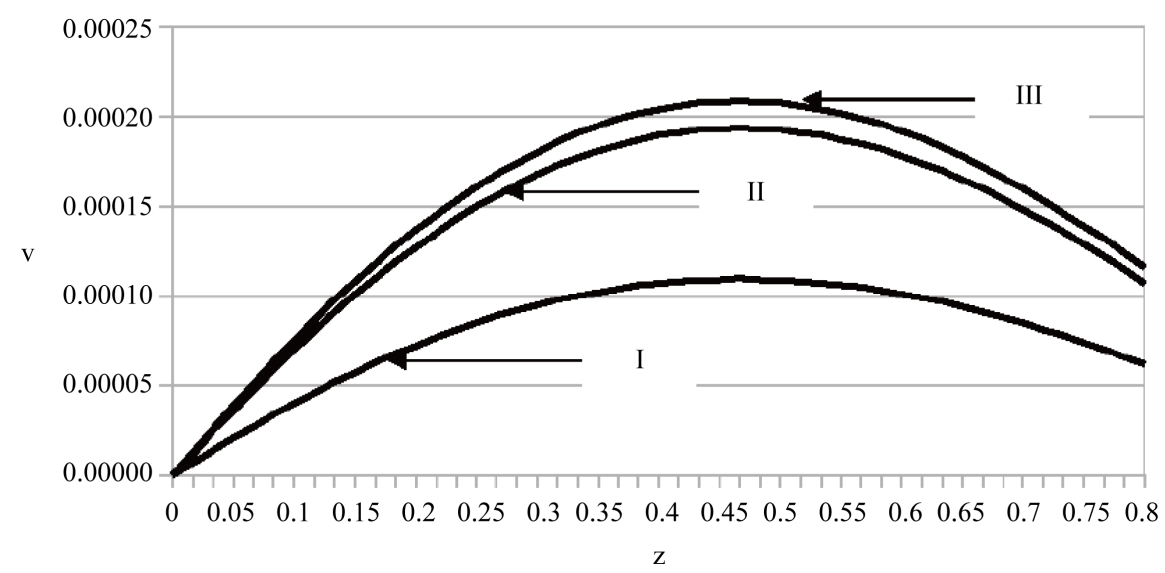

\begin{tabular}{|c|c|}
\hline Series & t \\
\hline I & 0.01 \\
\hline II & 0.10 \\
\hline III & 0.50 \\
\hline
\end{tabular}

Figure 9. Transient velocity profiles ( $v$ ) for $G r=10$.

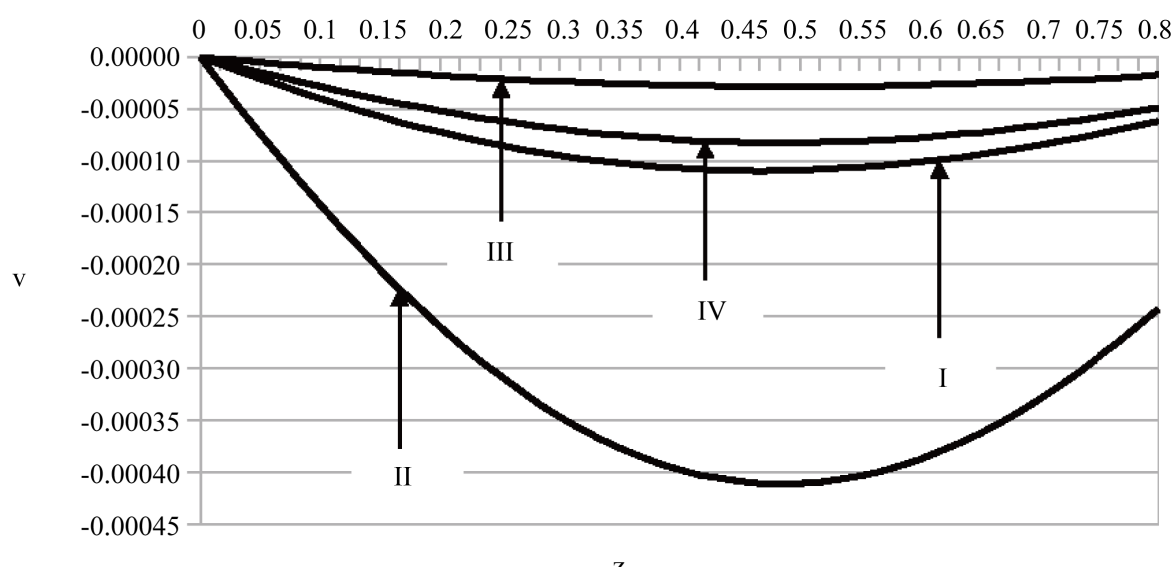

\begin{tabular}{|c|c|c|c|}
\hline Series & Pr & Er & R \\
\hline I & 0.71 & 0.001 & 3 \\
\hline II & 7 & 0.001 & 3 \\
\hline III & 0.71 & 0.005 & 3 \\
\hline IV & 0.71 & 0.001 & 30 \\
\hline
\end{tabular}

Figure 10. Transient velocity profiles $(v)$ for $G r=-10$.

3) there is an insignificant change in the transient primary velocity profile $(u)$ due to an increase in radiation parameter $(R)$.

From Figure 8 and Figure 9, for $G r>0$ (in the presence of cooling of the plate by free convection currents), it is observed that:

1) the transient secondary velocity profile $(v)$ increases due to an increase in time $(t)$ and Prandtl number $(P r)$;

$2)$ there is a fall in the transient secondary velocity profile $(v)$ due to an increase in rotation parameter $(E r)$ 


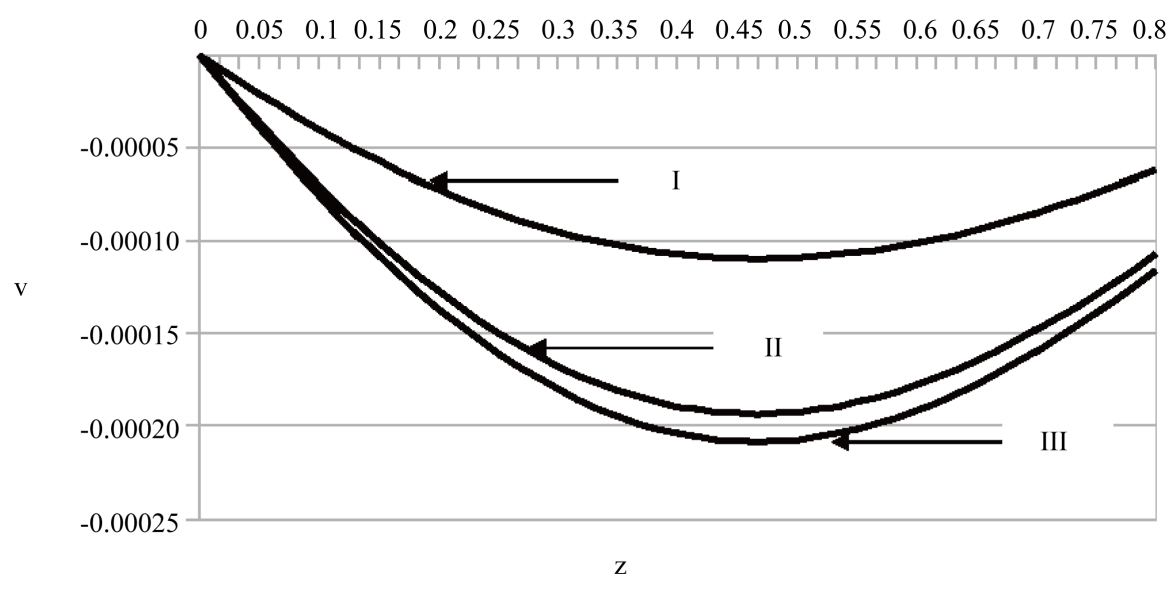

\begin{tabular}{|c|c|}
\hline Series & t \\
\hline I & 0.01 \\
\hline II & 0.10 \\
\hline III & 0.50 \\
\hline
\end{tabular}

Figure 11. Transient velocity profiles ( $v$ ) for $G r=-10$.

and radiation parameter $(R)$.

From Figure 10 and Figure 11, for $G r<0$ (in the presence of heating of the plate by free convection currents), it is observed that:

1) the secondary velocity profile $(v)$ is backward;

$2)$ there is a fall in the secondary velocity profile $(v)$ due to an increase in time $(t)$ and Prandtl number $(P r)$;

$3)$ there is a rise in the transient secondary velocity profile $(v)$ due to an increase in rotation parameter $(E r)$ and radiation parameter $(R)$.

\section{Conclusions}

In this work, a simulation was carried out on the hydromagnetic free convective flow past a vertical infinite porous plate in a rotating fluid. Very large temperatures were assumed in the analysis in order to make the radiative heat transfer significant. The velocity and the temperature fields were computed using a generic software tool based on the Nakamura finite difference scheme. From the results obtained, we observed that:

1) the development of generic tools can drastically simplify the solution of fluid flow problems;

2) only the input equations and the relevant parameters need to be defined in a generic file which will be used as input to the simulation system.

3 ) the postfix code is very efficient in the computation of arithmetic expressions.

\section{References}

[1] Greenspan, H.P. (1968) The Theory of Rotating Fluids. Cambridge University Press, Cambridge, UK

[2] Bachelor, G.K. (1970) An Introduction to Fluid Dynamics. Cambridge University Press, Cambridge, UK.

[3] Debnath, L. (1972) On Unsteady Magnetohydrodynamic Boundary Layers in a Rotating Fluid. Zeitschrift für Angewandte Mathematik und Mechanik, 52, 623-625. http://dx.doi.org/10.1002/zamm.19720521010

[4] Debnath, L. (1974) Resonant Oscillations of a Porous Plate in Electrically Conducting Fluid. Physics of Fluids, 17, 1704-1707. http://dx.doi.org/10.1063/1.1694959

[5] Debnath, L. (1975) Inertial Oscillations and Hydromagnetic Multiple Boundary Layers in a Rotating Fluid. Zeitschrift für Angewandte Mathematik und Mechanik, 55, 431-534. http://dx.doi.org/10.1002/zamm.19750550712

[6] Tokis, J.N. (1986) Unsteady MHD Free Convection Flows in a Rotating Fluid. Astrophysics and Space Science, 119, 305-313. 
[7] Naroua, H. (2010) Modeling of Unsteady MHD Free Convection Flow with Radiative Heat Transfer in a Rotating Fluid. Natural Science, 2, 1386-1393. http://dx.doi.org/10.4236/ns.2010.212169

[8] Yamauchi, J., Nakamura, S. and Nakano, H. (2000) Application of Modified Finite-Difference Formulas to the Analysis of z-Variant Rib Waveguides. IEEE Photonics Technology Letters, 12, 1001-1003. http://dx.doi.org/10.1109/68.867988

[9] Zhu, S., Yuan, G. and Sun, W. (2004) Convergence and Stability of Explicit/Implicit Schemes for Parabolic Equations with Discontinuous Coefficients. International Journal of Numerical Analysis and Modeling, 1, 131-145.

[10] Khader, M.M. and Ahmed, M.M. (2013) Numerical Simulation Using the Finite Difference Method for the Flow and Heat Transfer in a Thin Liquid Film over an Unsteady Stretching Sheet in a Saturated Porous Medium in the Presence of Thermal Radiation. Journal of King Saud University: Engineering Sciences, 25, 29-34. http://dx.doi.org/10.1016/j.jksues.2011.10.002

[11] Matsuoka, H. and Nakamura, K.-I. (2013) A Stable Finite Difference Method for a Cahn-Hilliard Type Equation with Long-Range Interaction. Science Reports of Kanazawa University, 57, 13-34.

[12] Cheng, P. (1964) Radiative Heat Transfer. American Institute of Aeronautics and Astronautics Journal, 2, $1664-1667$.

[13] Nakamura, S. (1991) Applied Numerical Methods with Software. Prentice-Hall International Editions, Upper Saddle River. 